work, because it allows antimicrobial susceptibilities (metronidazole, clarithromycine to be determined, and we would not replace it for a specific test that was not fully specific as suggested by the correspondents.

1 Veenendaal RA, Litchendahl-Bernards AT, Peña AS, et al. Influence of transport medium and transportation time on culture of Helicobacter pylori from gastric biopsies. $\mathcal{F}$ Clin Pathol 1993;46:561-3.

2 Peña AS, Endts HPh, Offerhaus CJA Hoogenboom-Verdegaal A, et al. Value of serology (ELISA and Immunoblotting) for the diagnosis of campylobacter pylori infection. Digestion 1989;44:131-41.

3 Lin SK, Lambert JR, Schembri $M$, et al. A comparison of diagnostic tests to determine Helicobacter pylori infection. $\mathcal{f}$ Gastroenterol Hepatol 1992;7:203-9.

4 Veldhuyzen van Zanten SJO, Tytgat KMAJ, de Gara CJ, et al. A prospective comparison de Gara CJ, et al. A prospective comparison
of symptoms and five diagnostic tests in patients with $H$ pylori positive and negative dyspepsia. Eur $\mathcal{f}$ Gastroenterol Hepatol 1991; 3:463-8.

5 Marshall BJ. Practical diagnosis of Helicobacter pylori. In: Marshal BJ, McCallum RW, Guerrant RL, eds. Helicobacter pylori in peptic ulceration and gastritis. Boston: Blackwell Scientific Publications 1991:139-59.

\section{Measurement of medical staff overload}

Dr Bignardi ${ }^{1}$ is correct in his conclusion that it is difficult to measure medical staff workload and requirements in microbiology departments. The current guidelines of the Royal College of Pathologists for consultant staffing suggest that for central laboratories in district general hospitals serving a population of approximately 250000 there should be at least two consultant medical microbiologists. ${ }^{2}$ A number of districts do not provide such staffing and cases need to be developed to persuade managers to provide appropriate cover. "Population served" is a crude measure of workload, even if referral patterns do not distort the picture. It is also clear that hospital bed numbers are not directly related to laboratory activity; indeed, for some hospitals reducing bed numbers has resulted in an increase in laboratory tests from outpatients, day cases, and GPs. Numbers of specimens and the number and nature of tests can be more closely related to laboratory activity and can be made more sophisticated by such systems as WELCAN, but these are not a measure of medical input; neither are they a measure of the quality of a microbiology service. Particular problems in measuring consultant microbiologist input are the contributions to core activities of the hospital(s) and clinics served-activities such as hospital infection control, policies for infected waste, chemical disinfection-and the general provision of advice on the management of infected patients. The latter aspects depend to a large extent on the case mix profile of the units served: intensive care units, special care baby units and oncology wards make particularly heavy demands on medical microbiologists. Although these matters are generally clear in principle, the allocation of numerical factors to reflect the workload has proved to be very difficult. Some of the problems of consultant staffing levels have been discussed in a recent article in $A C P$ $N_{e w s}{ }^{3}$ and the Microbiology Specialty Advisory Committee of the Royal College of Pathologists is currently examining this subject. It will not be easy to produce a universally acceptable measure, but the prob- lems must be addressed in order to try to achieve a composite workload definition that reflects the range of input required of a consultant microbiologist.

DR DAVIES Microbiology Specialist Advisory Committee, Royal College of Pathologists, 2 Carlton House Terrace, London SWIY $5 A F$

1 Bignardi GE. How many microbiologists are needed? $\mathcal{C}$ Clin Pathol 1993;46:1051-2.

2 Royal College of Pathologists. Medical and scientific staffing of national health service pathology departments. London: Roya College of Pathologists 1992:1-11.

3 Workload figures: whose norms are they anyway? ACP News 1993:11-12.

Dr Bignardi comments:

I welcome the interest by the Microbiology Specialist Advisory Committee of the Royal College of Pathologists: eliciting such interest was the main purpose of my report. In my opinion the current guidelines by the Royal College of Pathologists for consultant staffing in microbiology are so impractical that they cannot be implemented by the College itself. This is demonstrated by the fact that, during the period of my study, four job descriptions for single-handed consultants were approved by the College despite the fact that the respective populations exceeded 250000 (the College recommends two consultants for departments serving a population of approximately 250 000). According to $\mathrm{my}$ analysis, the case for a second full-time consultant microbiologist was very strong in two of these four hospitals.

One would hope that if a formula based on the weighted number of beds and specimens (and perhaps on other factors) was sanctioned and policed by the College, at the least the worst cases of understaffing could be eliminated. Since writing my report I have noticed some important trends: the overall number of both consultants and junior doctors in microbiology seems to be decreasing, many pathology departments have been asked to take substantial cuts in their budget over the next years, and the NHS Management Executive has commissioned a strategic review of pathology services which might throw the door open to more pathology privatisations.

Given the current political climate, I think it most important that we try to identify and quantify the minimum medical staff requirement for a good quality service in microbiology.

\section{Necrotising granulomas of the uterine corpus}

We read with interest the report by Drs Akosa and Boret of necrotising granulomas of the uterine corpus following Nd YAG laser ablation of the endometrium, ${ }^{1}$ and noted their reference to our original report of peritoneal granulomas following laser ablation. ${ }^{2}$

We subsequently reported the histological findings from four hysterectomy specimens obtained for various indications following Nd YAG laser ablation. ${ }^{3}$ Our findings were essentially the same as those of Akosa and Boret, and we were able to demonstrate by energy dispersive $x$-ray analysis that the black foreign material within the necrotising granulomas consisted largely of aluminium oxide compatible with the known composition of the sapphire laser probe.

We also provided evidence to support the hypothesis that recurrent bleeding following laser ablation is due to inspread of functional endometrium from the tubal ostia and isthmus, ${ }^{45}$ and were disappointed that Akosa and Boret made no comment on the histological appearances of the endometrium away from the obvious laser damage.

Finally, Akosa and Boret refer to the technique as endometrial resection which is in our view not correct, as the use of the $\mathrm{Nd}$ YAG laser is a technique for endometrial ablation.

JHF SMITH

A KENNEDY F SHARP Northern General Hospital,
NHS Trust, Herries Road,
Sheffield S5 $7 A U$ PC REID

Luton and Dunstable Hospital W THURRELI

University College Hospital,

1 Akosa AB, Boret F. Necrotising granulomas of the uterine corpus. F Clin Pathol 1993;46: 953-5.

2 Thurrell W, Reid PC, Kennedy A, Smith JHF. Necrotising granulomas of the peritoneum. Histopathology 1991;18:190

3 Reid PC, Thurrell W, Smith JHF, Kennedy A, Sharp F. Nd:YAG laser endometrial ablation: histological aspects of uterine healing. Int $\mathcal{f}$ Gynecol Pathol 1992;11:174-9.

4 Lomano JM. Photocoagulation of the endometrium with the Nd:YAG laser for the treatment of menorhagia: a report of te cases. F Reprod Med 1986;31:148-50.

5 Baggish MS, Baltoyannis P. New techniques for laser ablation of the endometrium in for laser ablation of the endometrium in 1988;159:287-92.

Drs Akosa and Boret comment:

We are grateful to Dr Smith et al for their prompt comment on our short report. This was basically intended to increase awareness among histopathologists of what has become a diagnostic quandary in the absence of adequate clinical information and in view of the increasing use of minimal invasive surgical techniques.

We noted in our report that the abnormalities in the endometrium were either complete or focal, the latter the cause of subsequent bleeding. The residual endometrium, although not stated in our report, was not confined only to the cornu as in the case referred to in the paper by Baggish and Baltoyannis. If one assumes that in every case of endometrial ablation the entire endometrium is destroyed, the hypothesis of inspread may be acceptable: in our experience this is not always the case.

Endometrial resection using laser and endometrial ablation have been and are used interchangeably. Our opening sentence which is now under discussion read, "Transcervical resection of the endometrium is a hysteroscopic method of endometrial ablation": this is self-explanatory.

Our literature search was confined to 1989 onwards, which explains why the papers by Baggish and Baltoyannis and Lomano were not cited. As for the paper by Reid et al, we can only assume that at the time of our search it had not been indexed. We have now read all these papers and they 